\title{
Adjuvant transarterial chemoembolization following radical resection for intrahepatic cholangiocarcinoma: $A$ multi-center retrospective study
}

\author{
Lei Wang ${ }^{*}$, Zi-Guo Lin ${ }^{2 *}$, Qiao Ke ${ }^{2 *}$, Jian-Ying Lou ${ }^{3}$, Shu-Guo Zheng, ${ }^{4}$ Xin-Yu Bi ${ }^{5}$, Jian-Ming Wang ${ }^{6}$, Wei \\ $\mathrm{Guo}^{7}, \mathrm{Fu}-\mathrm{Yu} \mathrm{Li}^{8}$, Jian Wang${ }^{9}$, Ya-Min Zheng ${ }^{10}$, Jing-Dong Li11, Shi Cheng ${ }^{12}$, Wei-Ping Zhou ${ }^{13}$, Yong-Yi \\ Zeng ${ }^{1,2 \bowtie}$
}

1. Department of Radiation Oncology, Mengchao Hepatobiliary Hospital of Fujian Medical University, Fuzhou China, 350025

2. Department of Hepatobiliary Surgery, Mengchao Hepatobiliary Hospital of Fujian Medical University, Fuzhou China, 350025

3. Department of hepatobiliary surgery, the Second Hospital affiliated to Zhejiang University, Hangzhou, China, 310009

4. Department of hepatobiliary surgery, the Southwest Hospital affiliated to the Army Medical University, Chongqing, China, 400038

5. Department of Hepatobiliary Surgery, Cancer Hospital, Chinese Academy of Medical Sciences, Beijing, China, 100021

6. Department of hepatobiliary surgery, Tongji Hospital affiliated to affiliated to Tongji Medical College, Huazhong University of Science \&Technology, Wuhan, Hubei, China, 430030

7. Department of Hepatobiliary Surgery, Beijing Friendship Hospital affiliated to Capital Medical University, Beijing, China, 100053

8. Department of Hepatobiliary Surgery, the West China Hospital of Sichuan University, Chengdu, China, 610041

9. Department of hepatobiliary surgery, Renji Hospital affiliated to Shanghai Jiaotong University, Shanghai, China, 200127

10. Department of Hepatobiliary Surgery, Xuanwu Hospital affiliated to Capital Medical University, Beijing, China, 100050

11. Department of Hepatobiliary Surgery, the affiliated Hospital of Chuanbei Medical University, Nanchong, China, 637000

12. Department of Hepatobiliary Surgery, Tiantan Hospital affiliated to Capital Medical University, Beijing, China, 100050

13. Department of Hepatobiliary Surgery III, Eastern Hepatobiliary Surgery Hospital, Secondary Military Medical University, Shanghai China, 200438

* Authors contributed equally in this study

$\triangle$ Corresponding authors: Yong-Yi Zeng and Wei-Ping Zhou. Mengchao Hepatobiliary Hospital of Fujian Medical University. Xihong Road 312, Fuzhou 350025, Fujian, PR, China. Tel: +86 13805083802; Fax: +86 05918370 3772; Email:lamp197311@126.com (Yong-Yi Zeng); ehphwp@126.com (Wei-Ping Zhou)

(c) The author(s). This is an open access article distributed under the terms of the Creative Commons Attribution License (https://creativecommons.org/licenses/by/4.0/). See http://ivyspring.com/terms for full terms and conditions.

Received: 2019.09.16; Accepted: 2020.03.27; Published: 2020.04.07

\begin{abstract}
Background and Aims: The prognosis of intrahepatic cholangiocarcinoma (ICC) after radical resection is far from satisfactory, but the effect of postoperative transarterial chemoembolization ( $p$-TACE) remains controversial. This multi-center retrospective study was to evaluate the clinical value of $\mathrm{p}$-TACE and identify the selected patients who would benefit from P-TACE.

Methods: Data of ICC patients who underwent radical resection with/without P-TACE therapy was obtained from 12 hepatobiliary centers in China between Jan 2014 and Jan 2017 . Overall survival (OS) was set as the primary endpoint, which was analyzed by the Kaplan-Meier method before and after propensity score matching (PSM). Subgroup analysis was conducted based on the established staging system and survival risk stratification.

Results: A total of 335 patients were enrolled in this study, including 39 patients in the P-TACE group and 296 patients in the non-TACE group. Median OS in the $\mathrm{P}$-TACE group was longer than that in the non-TACE group (63.0 months vs. 18.0 months, $P=0.041)$, which was confirmed after $1: 1 \quad P S M(P=0.009)$. According to the $8^{\text {th }}$ TNM staging system, patients with stage II and stage III stage would be benefited from $\mathrm{P}$-TACE $(P=0.021)$. Subgroup analysis stratified by risk factors showed that $\mathrm{P}$-TACE could only benefit patients with risk factors $<2(P=0.027)$.

Conclusion: Patients with ICC should be recommended to receive P-TACE following radical resection, especially for those with stage II, stage III or risk factors $<2$. However, the conclusion deserved further validation.
\end{abstract}

Key words: intrahepatic cholangiocarcinoma, transarterial chemoembolization, overall survival, propensity score matching 


\section{Introduction}

The incidence of intrahepatic cholangiocarcinoma (ICC) is increasing stably worldwide, which accounts for $10 \%-15 \%$ of primary liver cancers [1, 2]. The prognosis remains poor, partly because approximately half of the ICC patients have lost the chances of surgery at diagnosis [3, 4]. Currently, radical resection remains the most efficient strategy for ICC [5-8], but the 5-year overall survival (OS) after radical resection is $20 \%-35 \%[9,10]$. Hence, postoperative adjuvant treatments are badly warranted to improve the prognosis of ICC.

Transarterial chemoembolization (TACE) has been confirmed to be efficient in the improvement of prognosis of advanced and inoperable patients [11-13], but whether postoperative TACE (p-TACE) could benefit patients following radical resection remains controversial. The clinical value of $\mathrm{p}$-TACE for ICC has been evaluated in previously few studies [14-19], but it has yet reached a conclusion. However, randomized clinical trials (RCT) on this issue are rarely conducted mainly owing to the rare incidence of ICC. Hence, we collected the data from a multi-center retrospective study to evaluate the prognosis value of $\mathrm{p}$-TACE for patients with ICC receiving radical resection.

\section{Material and Methods}

\section{Patient selection}

This study was conducted to the ethical guideline of the 1975 Declaration of Helsinki and was approved by all the participating centers including Mengchao hepatobiliary hospital, Eastern hepatobiliary surgery hospital, Affiliated Cancer Hospital of Chinese Academy of Medical Sciences, Tongji Hospital, Beijing Friendship Hospital, Xuanwu Hospital, Tiantan Hospital, the affiliated Hospital of Chuanbei Medical University, Renji Hospital, the West China Hospital, the Southwest Hospital, and the Second Hospital of Zhejiang University. Informed consent was signed by all patients or their direct relatives before surgery. Data between Jan 2014 and Jan 2017 in the 12 centers were collected via electric case report form (CRF), including baseline characteristics, operation parameters, and tumor characteristics.

\section{Eligibility}

Patients were enrolled in this study if they 1) had a histopathologically confirmed diagnosis of ICC, 2) underwent radical resection with or without LND, 3) had no history of other malignancies, 4) had not received any preoperative anticancer therapy. Patients who had 1) incomplete clinical data, 2) preoperative obstructive jaundice, 3) extrahepatic metastasis, 4) mortality within one month after surgery, and 6) received other postoperative adjuvant therapies, such as radiotherapy, chemoradiotherapy, and immunotherapies were excluded from this study.

\section{Intervention}

A preoperative diagnosis of ICC was primarily based on radiological findings, with or without elevated CEA and CA19-9[2, 6], and liver biopsy was needed when the imaging features were not typical. The indications of surgical resection for ICC were as follows: 1) patients with performance status $0 \sim 1$ before surgery; 2) tumors with or without lymph node metastasis which were evaluated to be technically resectable; 3) Child-Pugh class A to B7; 4) the estimated volume of future liver remnant was $>30 \%$ in normal livers and $50 \%$ in cirrhotic livers; 5) patients without evidence of extrahepatic metastasis.

Radical resection was defined as a negative margin and without recurrence within two months after resection. All the hepatectomy and LND were conducted by highly experienced surgeons, although the procedures were a little different from each center in detail.

p-TACE was conducted only once within one to two months following resection according to the discussion of multiple discipline team. Briefly, chemotherapeutic agents including 5-fluorouracil (500 $\mathrm{mg})$, epirubicin $(20 \mathrm{mg})$ and hydroxycamptothecin (10 $\mathrm{mg}$ ) were injected into the predetermined hepatic artery using a 5-F catheter, and then an emulsion of lipiodol (5-10 mL) was inserted to embolize. Of note, patients who had: 1) an Eastern Cooperative Oncology Group (ECOG) score 0-1,2) Child-Pugh grade $\mathrm{A}$ or $\mathrm{B}, 3)$ normal kidney function, 4) white blood cell count $\geq 3.0 \times 10^{9} / \mathrm{L}$ and platelet count $\geq 50 \times$ $10^{9} / \mathrm{L}$ were eligible to receive $\mathrm{p}-\mathrm{TACE}$.

\section{Follow-up and definition of endpoints}

All patients were periodically followed up once every 2-3 months in the first 2 years and then once every 6 months. Routine follow-up items included liver function tests, serum levels of CA19-9, CEA and AFP, and abdominal ultrasound, and a contrast-enhanced CT or MRI was warranted once recurrence was clinically suspected. Recurrence or metastasis was defined as new lesions with radiologic characteristics of ICC, and further treatment was immediately adopted whenever recurrence was confirmed. The follow-up investigation was carried out until October 2018.

The primary endpoint was overall survival (OS), and the secondary endpoint was recurrence-free survival (RFS). OS was calculated from the resection 
to either the date of death or the latest follow-up. RFS was defined as the time from resection to the time of recurrence (either intrahepatic or extrahepatic) or the date of the latest follow-up.

\section{Propensity score matching}

Propensity score matching (PSM) was adopted to minify the selection bias [20], and the propensity score was determined using the potential confounding factors. Patients were then matched by a one-to-one ratio using the nearest neighbor method with a caliber of 0.2 .

\section{Statistics}

All the continuous variables were re-defined as categorical variables, hence all the variables were compared with the chi-square test or Fisher's exact test. Survival curves including OS and RFS were depicted using the Kaplan-Meier method and compared using the log-rank test. Risk factors associated with prognosis of ICC were determined by the forward method of the multivariate Cox regression model before and after PSM. Subgroup analysis was conducted based on the $8^{\text {th }}$ TNM staging system and risk factors.

Data analysis was conducted using SPSS 25.0, and PSM was conducted using RStudio. $\mathrm{P}<0.05$ in all cases was considered statistically significant.

\section{Results}

\section{Baseline characteristics}

Initially, 437 patients with ICC underwent radical resection, but 14 patients $(3.2 \%)$ were excluded for preoperative obstructive jaundice. After surgery, 13 patients $(3.0 \%)$ had died within one month, and 53 $(12.1 \%)$ patients received other postoperative adjuvant therapies. During the period of follow-up (1-73 months), 22 patients lost to follow-up. Finally, 335 patients were enrolled in this study, and 39 patients $(11.6 \%)$ received p-TACE within two months after surgery. Detailed were depicted in Fig 1.

The baseline characteristics of the 335 patients were shown in Table 1 . The median size of the resected tumor was $6.1 \mathrm{~cm}$, and 226 patients $(67.5 \%)$ had a single tumor. 76 patients $(22.7 \%)$ underwent LND, and LNM was confirmed by postoperative pathology in 41 patients (54.0\%). Before PSM, patients with age $<60$ years, ECOG score $<2$, and surgical margin $<1 \mathrm{~cm}$ were more likely to receive p-TACE (all $P<0.05$, Table 1), but the clinicopathological characteristics were comparable between the two groups after 1:1 PSM (all $P>0.05$, Table 1).

\section{Prognosis of patients treated with or without P-TACE in the overall cohort}

In the overall study population, the mean follow-up period was $21.5( \pm 3.0)$ months in the postoperative TACE group and $21.3( \pm 1.1)$ months in the non-TACE group. Median OS in the p-TACE group was longer than that in the non-TACE group (63.0 months vs. 18.0 months, $P=0.041$, Fig $2 A$ ). The $1-$, $2-$, and 3-year survival rates in the p-TACE group were higher than those in the non-TACE group $(76.9 \%$ vs. $65.9 \%, P=0.167 ; 66.7 \%$ vs. $46.0 \% ; P=0.015 ; 64.1 \%$ vs. $37.8 \% ; P=0.002$; respectively). Median RFS in the p-TACE group was longer than that in the non-TACE group (50.0 months vs. 10.0 months, $P=0.022$, Fig $2 B$ ). The 1-, 2-, and 3-year RFS rates in the p-TACE group in the p-TACE group and the non-TACE group were $61.5 \%$ vs. $45.3 \%(P=0.056) ; 56.4 \%$ vs. $31.8 \%(P=0.002)$; $56.4 \%$ vs. $25.7 \%(P<0.001)$; respectively.

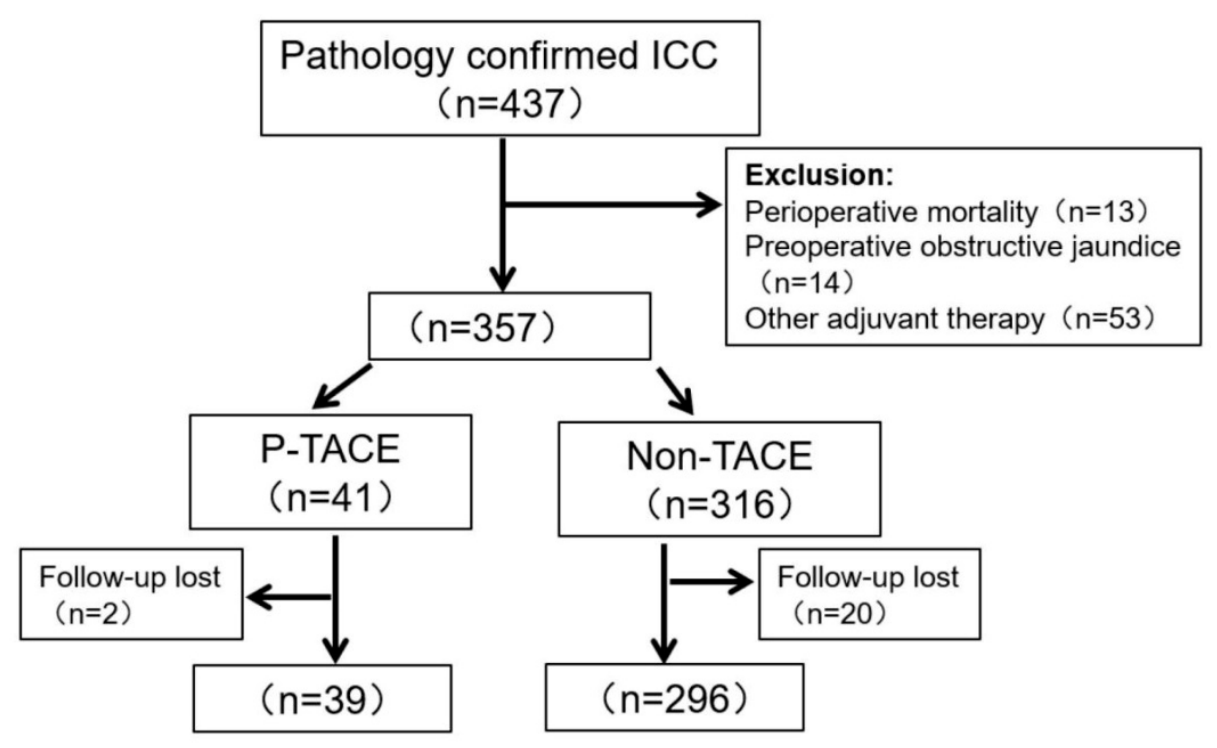

Fig 1. Flow chart of patients' enrollment 
After 1:1 PSM, median OS in the p-TACE group was longer than that in the non-TACE group (63.0 months vs. 18.0 months, $P=0.009$, Fig $2 C$ ). The 1-, 2-, and 3-year survival rates in the p-TACE group were significantly higher than those in the non-TACE group $(76.9 \%$ vs. $61.5 \%, P=0.141 ; 66.7 \%$ vs. $46.2 \%$; $P=0.068 ; \quad 64.1 \%$ vs. $35.9 \% ; P=0.013$; respectively). Median RFS in the p-TACE group was longer than that in the non-TACE group (50.0 months vs. 6.0 months, $P=0.004$, Fig 2D). The 1-, 2- and 3-year RFS rates in the p-TACE group were significantly higher than those in the non-TACE group $(61.5 \%$ vs. $35.9 \%$, $56.4 \%$ vs. $28.2 \%, 56.4 \%$ vs. $20.5 \%$, respectively, all $P<0.05)$.

\section{Risk factors associated with overall survival before and after PSM}

CA19-9 $\quad(\mathrm{HR}=1.458, \quad 95 \% \quad \mathrm{CI}=1.068 \sim 1.920$, $P=0.018), \quad$ LNM $\quad(\mathrm{HR}=1.897,95 \% \quad \mathrm{CI}=1.116 \sim 3.237$, $P=0.018)$, tumor size $(\mathrm{HR}=1.621,95 \% \mathrm{CI}=1.186 \sim 2.213$, $P=0.002)$, and satellite $(\mathrm{HR}=1.826,95 \% \mathrm{CI}=1.103$ 2.976, $P=0.019$ ) were identified as independent risk factors for OS in a whole cohort (Table 2). After 1:1 $\mathrm{PSM}$, tumor size $(\mathrm{HR}=2.121,95 \% \mathrm{CI}=1.123 \sim 4.011$, $P=0.021)$, satellite $(\mathrm{HR}=2.189,95 \% \mathrm{CI}=1.163 \sim 4.144$, $P=0.016) \quad$ and $\quad \mathrm{p}$-TACE $\quad(\mathrm{HR}=0.493, \quad 95 \%$ $\mathrm{CI}=0.264 \sim 0.911, \quad P=0.025) \quad$ were identified as independent risk factors for OS in a whole cohort (Table 3).

\section{Effect of p-TACE based on the 8th TNM staging system}

In the $8^{\text {th }} \mathrm{TNM}$ staging system, good prognostic stratification was observed among stage I, stage II and stage III $(\mathrm{P}<0.05$, Supplement Fig 1A). Considering patients in the stage II and stage III receiving p-TACE were too small, so we took them into one subgroup. Results showed that in the subgroup of patients with stage I, no significant difference was observed between $p$-TACE group and non-TACE group $(P=0.560$, Fig $3 \mathrm{~A})$; while in the subgroup of patients with stage II and stage III, significant difference was found between p-TACE group and non-TACE group $(P=0.021$, Fig 3B $)$.

Table 1. Baseline Characteristics before and after PSM

\begin{tabular}{|c|c|c|c|c|c|c|c|}
\hline & & \multicolumn{3}{|l|}{ Before PSM } & \multicolumn{3}{|l|}{ After PSM } \\
\hline & & Non-TACE & p-TACE & $P$-Value & Non-TACE & p-TACE & $P$-Value \\
\hline & & $(n=296)$ & $(n=39)$ & & $(n=39)$ & $(n=39)$ & \\
\hline \multirow[t]{2}{*}{ Gender } & Female & $110(37.2 \%)$ & $13(33.3 \%)$ & 0.772 & $10(25.6 \%)$ & $13(33.3 \%)$ & 0.619 \\
\hline & Male & $186(62.8 \%)$ & $26(66.7 \%)$ & & $29(74.4 \%)$ & $26(66.7 \%)$ & \\
\hline \multirow[t]{2}{*}{ Age } & $<60$ years & $176(59.5 \%)$ & $32(82.1 \%)$ & 0.011 & $32(82.1 \%)$ & $32(82.1 \%)$ & 1.000 \\
\hline & $\geq 60$ years & $120(40.5 \%)$ & $7(17.9 \%)$ & & $7(17.9 \%)$ & $7(17.9 \%)$ & \\
\hline \multirow[t]{2}{*}{ Hepatitis } & No & $193(65.2 \%)$ & $20(51.3 \%)$ & 0.128 & $25(64.1 \%)$ & $20(51.3 \%)$ & 0.359 \\
\hline & Yes & $103(34.8 \%)$ & $19(48.7 \%)$ & & $14(35.9 \%)$ & $19(48.7 \%)$ & \\
\hline \multirow[t]{2}{*}{ ECOG grade } & $<2$ & $236(79.7 \%)$ & $37(94.9 \%)$ & 0.039 & $38(97.4 \%)$ & $37(94.9 \%)$ & 1.000 \\
\hline & $\geq 2$ & $60(20.3 \%)$ & $2(5.1 \%)$ & & $1(2.6 \%)$ & $2(5.1 \%)$ & \\
\hline \multirow[t]{2}{*}{ CA19-9 } & $\leq 37 \mathrm{U} / \mathrm{L}$ & $213(72.0 \%)$ & $26(66.7 \%)$ & 0.618 & $24(61.5 \%)$ & $26(66.7 \%)$ & 0.813 \\
\hline & $>37 \mathrm{U} / \mathrm{L}$ & $83(28.0 \%)$ & $13(33.3 \%)$ & & $15(38.5 \%)$ & $13(33.3 \%)$ & \\
\hline \multirow[t]{2}{*}{ TBil } & $\leq 20 \mu \mathrm{mol} / \mathrm{L}$ & $155(52.4 \%)$ & $18(46.2 \%)$ & 0.576 & $13(33.3 \%)$ & $18(46.2 \%)$ & 0.355 \\
\hline & $>20 \mu \mathrm{mol} / \mathrm{L}$ & $141(47.6 \%)$ & $21(53.8 \%)$ & & $26(66.7 \%)$ & $21(53.8 \%)$ & \\
\hline \multirow[t]{2}{*}{ Child-Pugh } & A & $187(63.2 \%)$ & $28(71.8 \%)$ & 0.380 & $30(76.9 \%)$ & $28(71.8 \%)$ & 0.795 \\
\hline & B & $109(36.8 \%)$ & $11(28.2 \%)$ & & $9(23.1 \%)$ & $11(28.2 \%)$ & \\
\hline \multirow[t]{2}{*}{ Blood loss } & $\leq 400 \mathrm{~mL}$ & $244(82.4 \%)$ & $35(89.7 \%)$ & 0.357 & $30(76.9 \%)$ & $35(89.7 \%)$ & 0.224 \\
\hline & $>400 \mathrm{~mL}$ & $52(17.6 \%)$ & $4(10.3 \%)$ & & $9(23.1 \%)$ & $4(10.3 \%)$ & \\
\hline \multirow[t]{2}{*}{ Transfusion } & No & $260(87.8 \%)$ & $36(92.3 \%)$ & 0.581 & $30(76.9 \%)$ & $36(92.3 \%)$ & 0.117 \\
\hline & Yes & $36(12.2 \%)$ & $3(7.7 \%)$ & & $9(23.1 \%)$ & $3(7.7 \%)$ & \\
\hline \multirow[t]{2}{*}{ Margin } & Wide & $69(23.3 \%)$ & $18(46.2 \%)$ & 0.004 & $18(46.2 \%)$ & $18(46.2 \%)$ & 1.000 \\
\hline & Narrow & $227(76.7 \%)$ & $21(53.8 \%)$ & & $21(53.8 \%)$ & $21(53.8 \%)$ & \\
\hline \multirow[t]{2}{*}{ Differentiation } & Well \&Moderate & $247(83.4 \%)$ & $34(87.2 \%)$ & 0.716 & $28(71.8 \%)$ & $34(87.2 \%)$ & 0.161 \\
\hline & Poor & $49(16.6 \%)$ & $5(12.8 \%)$ & & $11(28.2 \%)$ & $5(12.8 \%)$ & \\
\hline \multirow[t]{2}{*}{ Tumor Number } & Single & $199(67.2 \%)$ & $27(69.2 \%)$ & 0.945 & $28(71.8 \%)$ & $27(69.2 \%)$ & 1.000 \\
\hline & Multiple & $97(32.8 \%)$ & $12(30.8 \%)$ & & $11(28.2 \%)$ & $12(30.8 \%)$ & \\
\hline \multirow[t]{2}{*}{ Tumor size } & $\leq 5 \mathrm{~cm}$ & $101(34.1 \%)$ & $21(53.8 \%)$ & 0.026 & $21(53.8 \%)$ & $21(53.8 \%)$ & 1.000 \\
\hline & $>5 \mathrm{~cm}$ & $195(65.9 \%)$ & $18(46.2 \%)$ & & $18(46.2 \%)$ & $18(46.2 \%)$ & \\
\hline \multirow[t]{2}{*}{ Satellite } & No & $204(68.9 \%)$ & $29(74.4 \%)$ & 0.611 & $30(76.9 \%)$ & $29(74.4 \%)$ & 1.000 \\
\hline & Yes & $92(31.1 \%)$ & $10(25.6 \%)$ & & $9(23.1 \%)$ & $10(25.6 \%)$ & \\
\hline \multirow[t]{2}{*}{ Neurological invasion } & No & $277(93.6 \%)$ & $38(97.4 \%)$ & 0.551 & $33(84.6 \%)$ & $38(97.4 \%)$ & 0.113 \\
\hline & Yes & $19(6.4 \%)$ & $1(2.6 \%)$ & & $6(15.4 \%)$ & $1(2.6 \%)$ & \\
\hline \multirow[t]{2}{*}{ LNM } & No & $261(88.2 \%)$ & $33(84.6 \%)$ & 0.706 & $34(87.2 \%)$ & $33(84.6 \%)$ & 1.000 \\
\hline & Yes & $35(11.8 \%)$ & $6(15.4 \%)$ & & $5(12.8 \%)$ & $6(15.4 \%)$ & \\
\hline \multirow[t]{2}{*}{ MVI } & No & $268(90.5 \%)$ & $37(94.9 \%)$ & 0.554 & $37(94.9 \%)$ & $37(94.9 \%)$ & 1.000 \\
\hline & Yes & $28(9.5 \%)$ & $2(5.1 \%)$ & & $2(5.1 \%)$ & $2(5.1 \%)$ & \\
\hline \multirow[t]{2}{*}{ AJCC } & I & $169(57.1 \%)$ & $24(61.5 \%)$ & 0.547 & $23(59.0 \%)$ & $24(61.5 \%)$ & 0.855 \\
\hline & II-III & $127(42.9 \%)$ & $15(38.5 \%)$ & & $16(241.0 \%)$ & $9(38.5 \%)$ & \\
\hline
\end{tabular}

Abbreviations: PSM, propensity score matching; ECOG, the Eastern Cooperative Oncology Group; TB, total bilirubin; LNM, lymph node metastasis; MVI, microvascular invasion; AJCC, American joint committee on cancer staging; p-TACE, postoperative transarterial chemoembolization. 
^
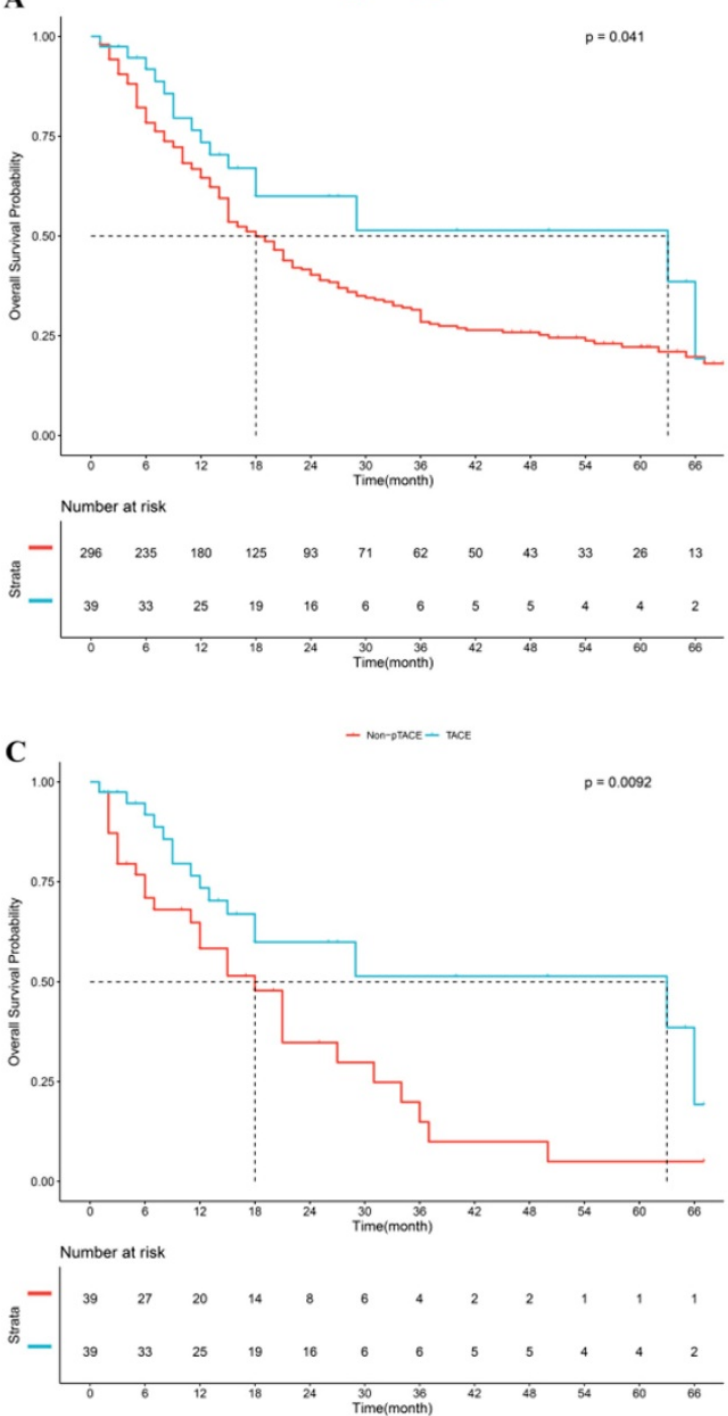
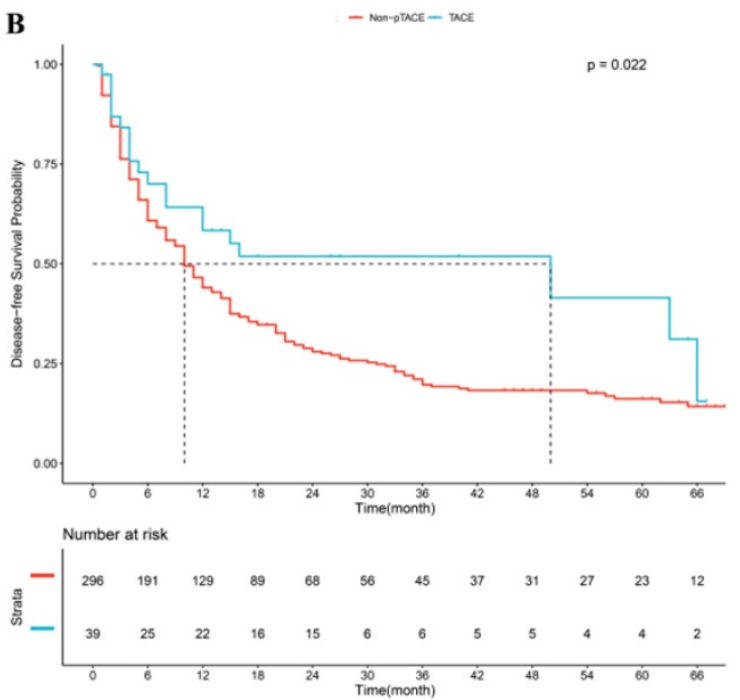

D
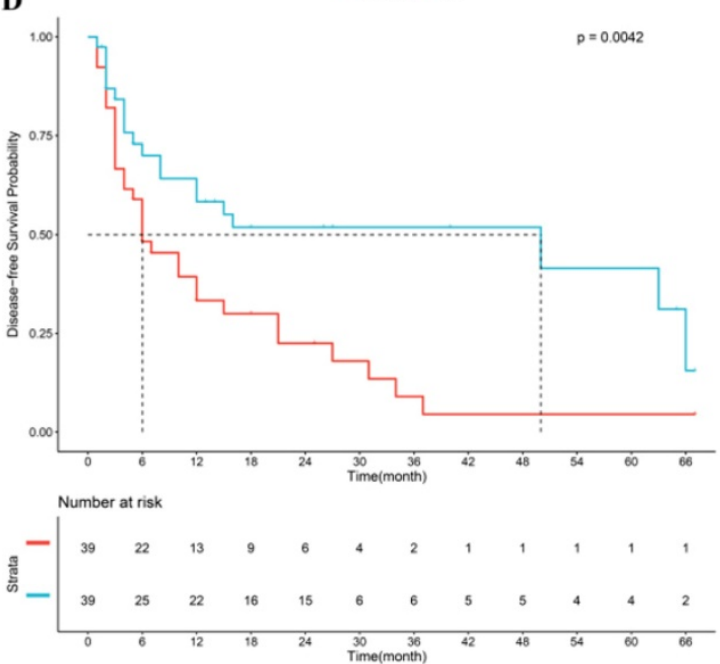

Fig 2. Kaplan-Meier analysis of overall survival (A) and recurrence-free survival (B) in whole cohort, Kaplan-Meier analysis of overall survival (C) and recurrence-free survival (D) after propensity score matching

Table 2. Univariate and multivariate analysis of overall survival for patients with intrahepatic cholangiocarcinoma in a whole cohort

\begin{tabular}{|c|c|c|c|c|c|c|}
\hline & \multicolumn{3}{|c|}{ Univariate analysis } & \multicolumn{3}{|c|}{ Multivariate analysis } \\
\hline & $\overline{\text { HR }}$ & $95 \% \mathrm{CI}$ & $P$ & HR & $95 \% \mathrm{CI}$ & $P$ \\
\hline Gender (Female/ Male) & 1.244 & $0.935-1.655$ & 0.134 & & & \\
\hline Age ( $<60$ years vs $\geq 60$ years) & 1.133 & $0.862-1.490$ & 0.370 & & & \\
\hline Hepatitis (No vs Yes) & 0.874 & $0.659-1.159$ & 0.349 & & & \\
\hline ECOG grade $(<2$ vs $\geq 2)$ & 1.342 & $0.969-1.858$ & 0.077 & & & \\
\hline CA19-9 ( $\leq 37 \mathrm{U} / \mathrm{L}$ vs $>37 \mathrm{U} / \mathrm{L})$ & 1.549 & $1.158-2.075$ & 0.003 & 1.458 & $1.068-1.920$ & 0.018 \\
\hline TBil $(\leq 20 \mu \mathrm{mol} / \mathrm{L}$ vs $>20 \mu \mathrm{mol} / \mathrm{L})$ & 0.855 & $0.653-1.120$ & 0.256 & & & \\
\hline Child-Pugh (A vs B) & 1.049 & $0.786-1.375$ & 0.755 & & & \\
\hline Blood loss $(\leq 400 \mathrm{~mL}$ vs $>400 \mathrm{~mL})$ & 1.158 & $0.805-1.656$ & 0.429 & & & \\
\hline Transfusion (No vs Yes) & 1.360 & $0.907-2.039$ & 0.136 & & & \\
\hline Margin (Wide vs Narrow) & 1.318 & $0.945-1.829$ & 0.097 & & & \\
\hline Differentiation (Well \&moderate vs Poor) & 1.258 & $0.868-1.815$ & 0.224 & & & \\
\hline Tumor number (Single vs Multiple) & 1.658 & $1.245-2.187$ & $<0.001$ & & & \\
\hline Tumor size $(\leq 5 \mathrm{~cm}$ vs $>5 \mathrm{~cm})$ & 1.729 & $1.276-2.315$ & $<0.001$ & 1.621 & $1.186-2.213$ & 0.002 \\
\hline Satellite (No vs Yes) & 1.946 & $1.468-2.588$ & $<0.001$ & 1.826 & $1.103-2.976$ & 0.019 \\
\hline Neurological invasion (No vs Yes) & 1.231 & $0.715-2.120$ & 0.453 & & & \\
\hline LNM (No vs Yes) & 1.905 & $1.282-2.831$ & 0.001 & 1.897 & $1.116-3.237$ & 0.018 \\
\hline MVI (No vs Yes) & 1.515 & $0.978-2.336$ & 0.065 & & & \\
\hline AJCC (I vs II-III) & 1.648 & $1.226-2.227$ & 0.001 & & & \\
\hline p-TACE (No vs Yes) & 0.597 & $0.358-0.994$ & 0.047 & & & \\
\hline
\end{tabular}


Table 3. Univariate and multivariate analysis of overall survival for patients with intrahepatic cholangiocarcinoma after propensity score matching

\begin{tabular}{|c|c|c|c|c|c|c|}
\hline & \multicolumn{3}{|c|}{ Univariate analysis } & \multicolumn{3}{|c|}{ Multivariate analysis } \\
\hline & HR & $95 \% \mathrm{CI}$ & $P$ & HR & $95 \% \mathrm{CI}$ & $P$ \\
\hline Gender (female/male) & 1.642 & $0.786-3.387$ & 0.179 & & & \\
\hline Age ( $<60$ years vs $\geq 60$ years) & 1.392 & $0.638-3.021$ & 0.411 & & & \\
\hline Hepatitis (No vs Yes) & 1.221 & $0.662-2.263$ & 0.522 & & & \\
\hline ECOG grade $(<2$ vs $\geq 2)$ & 0.373 & $0.051-2.711$ & 0.328 & & & \\
\hline CA19-9 ( $\leq 37 \mathrm{U} / \mathrm{L}$ vs $>37 \mathrm{U} / \mathrm{L})$ & 1.958 & $1.051-3.567$ & 1.842 & & & \\
\hline TBil $(\leq 20 \mu \mathrm{mol} / \mathrm{L}$ vs $>20 \mu \mathrm{mol} / \mathrm{L})$ & 0.656 & $0.364-1.211$ & 0.176 & & & \\
\hline Child-Pugh (A vs B) & 1.011 & $0.513-1.989$ & 0.978 & & & \\
\hline Blood loss $(\leq 400 \mathrm{~mL}$ vs $>400 \mathrm{~mL})$ & 1.243 & $0.514-2.998$ & 0.632 & & & \\
\hline Transfusion (No vs Yes) & 1.551 & $0.684-3.53$ & 0.294 & & & \\
\hline Margin (Wide vs Narrow) & 0.911 & $0.489-1.656$ & 0.752 & & & \\
\hline Differentiation (Well \&moderate vs Poor) & 2.042 & $0.956-4.325$ & 0.063 & & & \\
\hline Tumor Number (Single vs Multiple) & 1.222 & $0.643-2.321$ & 0.543 & & & \\
\hline Tumor size $(\leq 5 \mathrm{~cm}$ vs $>5 \mathrm{~cm})$ & 1.986 & $1.068-3.737$ & 0.031 & 2.121 & $1.123-4.011$ & 0.021 \\
\hline Satellite (No vs Yes) & 2.387 & $1.278-4.465$ & 0.006 & 2.189 & $1.163-4.144$ & 0.016 \\
\hline Neurological invasion (No vs Yes) & 2.285 & $0.947-5.512$ & 0.064 & & & \\
\hline LNM (No vs Yes) & 1.312 & $0.514-3.368$ & 0.578 & & & \\
\hline MVI (No vs Yes) & 0.662 & $0.158-2.812$ & 0.578 & & & \\
\hline AJCC (I vs II-III) & 0.889 & $0.442-1.816$ & 0.757 & & & \\
\hline p-TACE (No vs Yes) & 0.438 & $0.241-0.834$ & 0.011 & 0.493 & $0.264-0.911$ & 0.025 \\
\hline
\end{tabular}
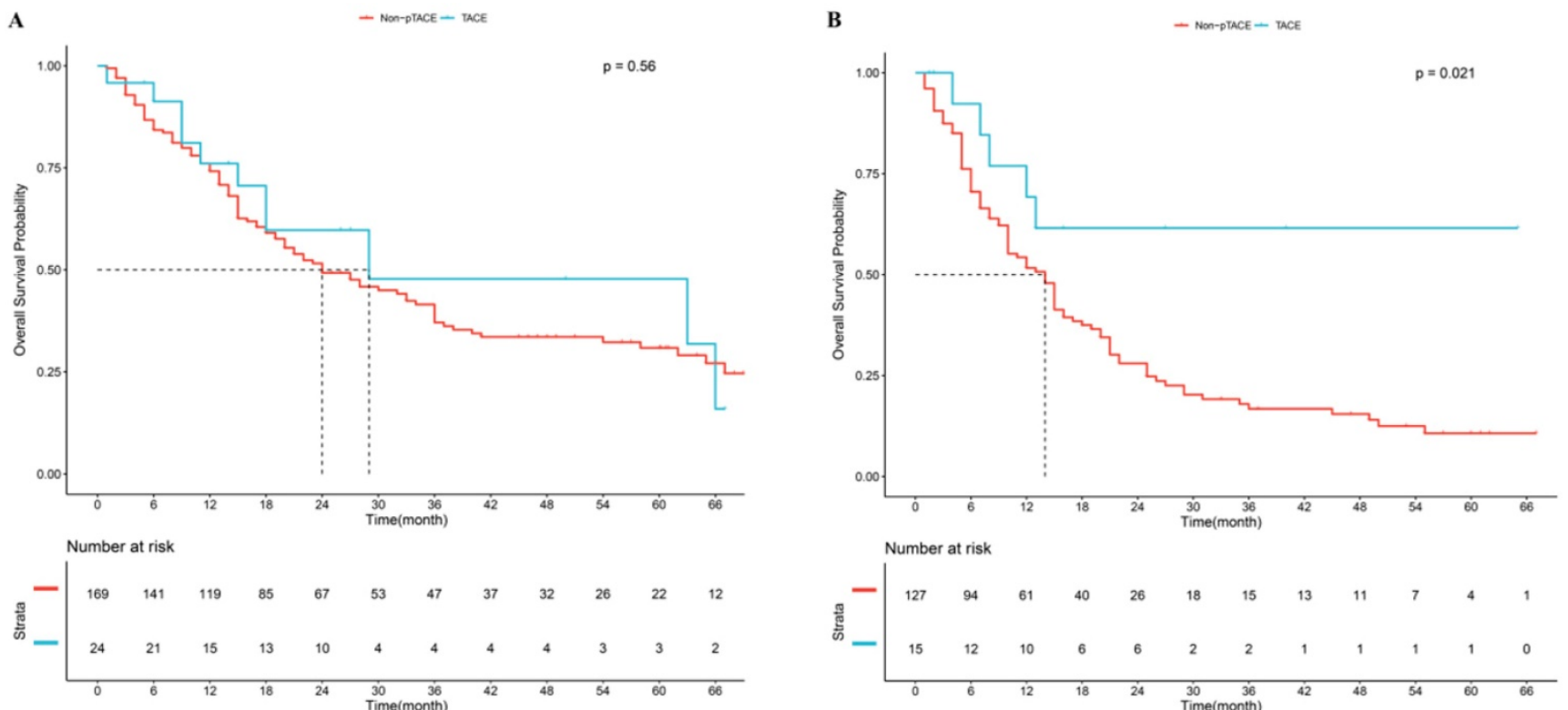

Fig 3. Kaplan-Meier analysis of overall survival based on $8^{\text {th }}$ AJCC staging system. (A), subgroup of patients with stage I, (B) subgroup of patients with stage II and stage III

\section{Effect of p-TACE based on risk factors}

CA19-9, LNM, tumor size, and satellite were confirmed to be independent risk factors of OS, and patients were divided into "high risk" and "low risk" subgroups according to the number of risk factors. Results showed that good prognostic stratification was observed between patients with risk factors $<2$ and patients with risk factors $\geq 2(P<0.05$, Supplement Fig 1B). Further analysis showed that in the subgroup of patients with risk factors $<2$, significant difference was observed between p-TACE group and non-TACE group $(P=0.027$, Fig $4 \mathrm{~A})$; while in the subgroup of patients with risk factors $\geq 2$, no significant difference was found between $\mathrm{p}$-TACE group and non-TACE group $(P=0.840$, Fig $4 \mathrm{~B})$.

\section{Discussion}

The prognosis of ICC after radical resection remains poor $[5,7,21]$, and strategies intended to reduce early recurrence and improve the long-term prognosis are still badly warranted. p-TACE has been tried with the aim of anti-recurrence, but its efficacy remains controversial[14-19]. In this study, 39 of 335 patients $(11.6 \%)$ received p-TACE following radical resection, which was lower than that in the previous reports $[16,18]$. Results showed that patients in the p-TACE group enjoyed longer median OS and RFS 
A

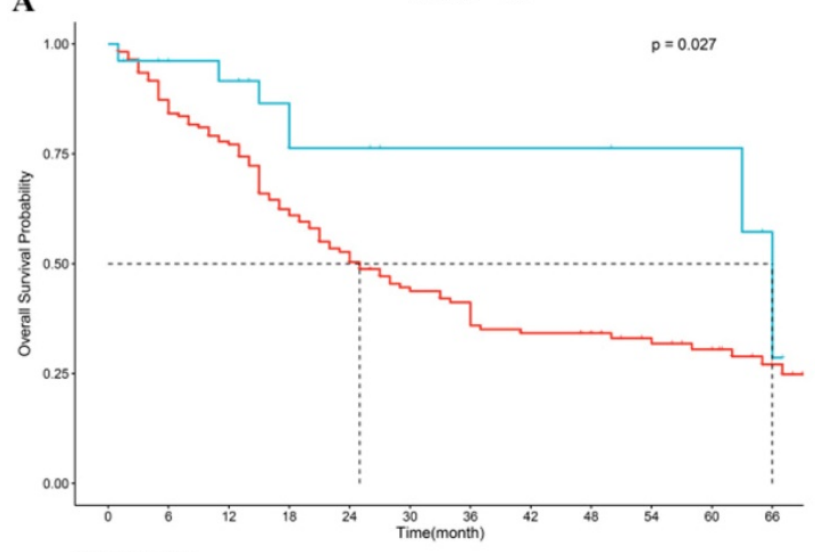

\begin{tabular}{|cccccccccccc} 
Number at risk \\
169 & 141 & 119 & 87 & 68 & 53 & 47 & 38 & 35 & 27 & 23 & 13 \\
26 & 22 & 20 & 17 & 14 & 5 & 5 & 5 & 5 & 4 & 4 & 2 \\
\hline 0 & 6 & 12 & 18 & 24 & $\begin{array}{c}30 \\
\text { Time(month) }\end{array}$ & 42 & 48 & 54 & 60 & 66
\end{tabular}
B
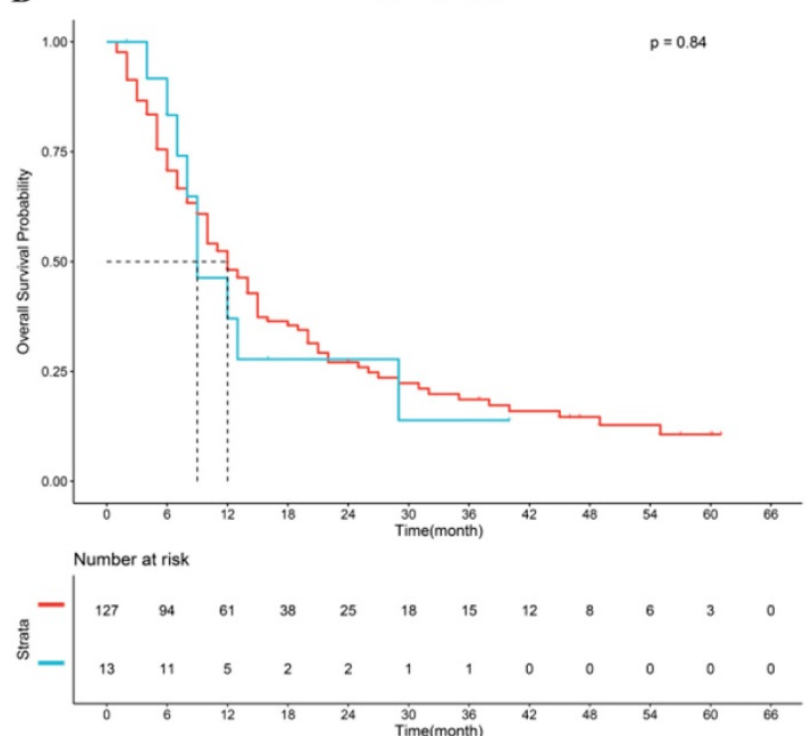

Fig 4. Kaplan-Meier analysis of overall survival based on risk factors. (A), subgroup of patients with "low risk", (B) subgroup of patients with "high risk".

than those in the non-TACE group before and after PSM (all P<0.05). Hence, TACE should be conducted after radical resection for ICC.

Radical resection is still the first-line treatment for patients with ICC [5-7], although half of the patients have lost the chances of resection at diagnosis $[3,4]$. However, the median OS of ICC after radical resection has been reported to be 21.0-39.0 months [10, $22,23]$, which is far from satisfactory. Reasons might be as follows: 1) aggressive characteristics of ICC [24], 2) high incidence of LNM but low incidence of LND $[25,26]$, and 3) high rate of early recurrence [27, 28]. In this study, the incidences of LND and LNM were $22.7 \%$, and $54.0 \%$, respectively, and the rate of recurrence within two years after radical resection was $61.5 \%$. Hence, more strategies should be considered to improve the prognosis of ICC.

TACE is often considered as one of the important postoperative adjuvant therapies for primary liver cancers $[29,30]$, and has been conducted prevalently worldwide [31, 32]. Currently, few studies reported the clinical value of p-TACE for ICC [14-19], but conclusion has yet to be reached. In this study, the benefit of $\mathrm{p}$-TACE group was observed in the whole cohort $(P<0.05)$, and it was confirmed after 1:1 PSM $(P<0.05)$, which indicated that our results were very convincing.

However, one size was not fit for all. Previously, only patients with advanced stage or scores $\geq 77$ based on the established ICC nomogram were reported to be benefited from p-TACE $[16,19]$. In this study, we found that only patients with stage II and stage III according to the $8^{\text {th }}$ TNM staging system would be benefited from p-TACE, which was consistent with previous reports. However, query remains, are patients with "high risk" benefited from prophylactic p-TACE? In this study, subgroup analysis showed that only patients with risk factors $<2$ would be benefited from $\mathrm{p}$-TACE, rather than those with risk factors $\geq 2$. In our opinion, patients with "high risk" were more likely to relapse, and need more aggressive strategies.

\section{Limitations}

Nevertheless, there were several restrictions in this study. First, it was a retrospective study, and recalling bias was inevitable. Second, confounding factors related to the efficacy of $\mathrm{p}$-TACE were almost inevitable, although a well-designed PSM was carried out. Thirdly, the incidence of patients receiving p-TACE was low $(39 / 335,11.6 \%)$, which was not optimal to reach a robust conclusion. The last but not the least, patients receiving p-TACE were typical present with aggressive characteristics and/or not sensitive to chemotherapy.

\section{Conclusion}

In summary, p-TACE would benefit patients with ICC receiving radical resection, especially for those with stage II, stage III or risk factors $<2$. However, the conclusion requires further validation.

\section{Abbreviations}

ICC, intrahepatic cholangiocarcinoma; TACE, transarterial chemoembolization; PSM, propensity score matching; LND, lymph node dissection; LNM, lymph node metastasis; OS, overall survival; RFS, 
recurrence-free survival; HR, Hazard ratio; OR, odd ratio; $\mathrm{CI}$, confidence interval.

\section{Supplementary Material}

Supplementary figure.

http://www.jcancer.org/v11p4115s1.pdf

\section{Acknowledgments}

Thank for the subsidy of Fujian provincial medical center of hepatobiliary.

\section{Competing Interests}

The authors have declared that no competing interest exists.

\section{References}

1. Ferlay J, Colombet M, Soerjomataram I, et al. Estimating the global cancer incidence and mortality in 2018: GLOBOCAN sources and methods. Int J Cancer. 2019; 144(8): 1941-53

2. Rd BA, D'Angelica MI, Abbott DE, et al. NCCN Guidelines Insights: Hepatobiliary Cancers, Version 1.2017. J Natl Compr Canc Netw. 2017; 15(5): $563-73$

3. Amini N, Ejaz A, Spolverato G, et al. Temporal trends in liver-directed therapy of patients with intrahepatic cholangiocarcinoma in the United States: a population-based analysis. J Surg Oncol. 2014; 110: 163-70.

4. Weber SM, Jarnagin WR, Klimstra D, et al. Intrahepatic cholangiocarcinoma: resectability, recurrence pattern, and outcomes. J Am Coll Surg. 2001; 193: 384-91.

5. Wang $\mathrm{K}$, Zhang $\mathrm{H}$, Xia $\mathrm{Y}$, et al. Surgical options for intrahepatic cholangiocarcinoma. Hepatobiliary Surg Nutr. 2017; 6: 79-90.

6. Weber SM, Ribero D, O'Reilly EM, et al. Intrahepatic Cholangiocarcinoma: expert consensus statement. HPB. 2015; 17: 669-80.

7. Waisberg DR, Pinheiro RS, Nacif LS, et al. Resection for intrahepatic cholangiocellular cancer: new advances. Transl Gastroenterol Hepatol. 2018; 3 : 60 .

8. Shen F, Xie ZH, Xia Y, et al. Progress on surgical treatment of intrahepatic cholangiocarcinoma. Zhonghua Wai Ke Za Zhi. 2019;57: 241-6.

9. Si A, Li J, Xiang H, et al. Actual over 10-year survival after liver resection for patients with intrahepatic cholangiocarcinoma. Oncotarget. 2017; 8: 44521-32.

10. Zhang Y, Shi S, Yang H, et al. Systemic inflammation score predicts survival in patients with intrahepatic cholangiocarcinoma undergoing curative resection. J Cancer. 2019; 10: 494-503.

11. Konstantinidis IT, Groot KB, Do RK, et al. Unresectable intrahepatic cholangiocarcinoma: Systemic plus hepatic arterial infusion chemotherapy is associated with longer survival in comparison with systemic chemotherapy lone. Cancer-Am Cancer Soc. 2016; 122: 758-65.

12. Savic LJ, Chapiro J, Geschwind JH. Intra-arterial embolotherapy for intrahepatic cholangiocarcinoma: update and future prospects. Hepatobiliary Surg Nutr. 2017: 6: 7-21.

13. Boehm LM, Jayakrishnan TT, Miura JT, et al. Comparative effectiveness of hepatic artery based therapies for unresectable intrahepatic cholangiocarcinoma. J Surg Oncol. 2015; 111: 213-20.

14. Jeong S, Zheng B, Wang J, et al. Transarterial Chemoembolization: A Favorable Postoperative Management to Improve Prognosis of Hepatitis B Virus-associated Intrahepatic Cholangiocarcinoma after Surgical Resection. Int J Biol Sci. 2017; 13: 1234-41.

15. Lu Z, Liu S, Yi Y, et al. Serum gamma-glutamyl transferase levels affect the prognosis of patients with intrahepatic cholangiocarcinoma who receive postoperative adjuvant transcatheter arterial chemoembolization: A propensity score matching study. Int J Surg. 2017; 37: 24-8

16. Li J, Wang Q, Lei Z, et al. Adjuvant Transarterial Chemoembolization Following Liver Resection for Intrahepatic Cholangiocarcinoma Based on Survival Risk Stratification. Oncologist. 2015; 20: 640-7.

17. Li T, Qin LX, Zhou J, et al. Staging, prognostic factors and adjuvant therapy of intrahepatic cholangiocarcinoma after curative resection. Liver Int. 2014; 34: 953-60.

18. Shen WF, Zhong $\mathrm{W}$, Liu $\mathrm{Q}$, et al. Adjuvant transcatheter arterial chemoembolization for intrahepatic cholangiocarcinoma after curative surgery: retrospective control study. World J Surg. 2011; 35: 2083-91.

19. Wu ZF, Zhang HB, Yang N, et al. Postoperative adjuvant transcatheter arterial chemoembolisation improves survival of intrahepatic cholangiocarcinoma patients with poor prognostic factors: results of a large monocentric series. Eur Surg Oncol. 2012; 38: 602-10.

20. Stuart EA, Lee BK, Leacy FP. Prognostic score-based balance measures can be a useful diagnostic for propensity score methods in comparative effectiveness research. J Clin Epidemiol. 2013; 66: S84-90.
21. $\mathrm{Si} \mathrm{A}$, Li J, Xiang $\mathrm{H}$, et al. Actual over 10-year survival after liver resection for patients with intrahepatic cholangiocarcinoma. Oncotarget. 2017; 8: 44521-32.

22. Wang $\mathrm{Y}, \mathrm{Li} \mathrm{J}$, Xia $\mathrm{Y}$, et al. Prognostic nomogram for intrahepatic cholangiocarcinoma after partial hepatectomy. J Clin Oncol. 2013; 31: 1188-95.

23. Sasaki K, Margonis GA, Andreatos N, et al. Preoperative Risk Score and Prediction of Long-Term Outcomes after Hepatectomy for Intrahepatic Cholangiocarcinoma. J Am Coll Surg. 2018; 226: 393-403.

24. Zhou XD, Tang ZY, Fan J, et al. Intrahepatic cholangiocarcinoma: report of 272 patients compared with 5,829 patients with hepatocellular carcinoma. J Cancer Res Clin Oncol. 2009; 135: 1073-80.

25. Zhang XF, Chakedis J, Bagante F, et al. Trends in use of lymphadenectomy in surgery with curative intent for intrahepatic cholangiocarcinoma. Br J Surg. 2018; 105: 857-66.

26. Clark CJ, Wood-Wentz CM, Reid-Lombardo KM, et al. Lymphadenectomy in the staging and treatment of intrahepatic cholangiocarcinoma: a population-based study using the National Cancer Institute SEER database. HPB (Oxford). 2011; 13: 612-20.

27. Hu LS, Zhang XF, Weiss M, et al. Recurrence Patterns and Timing Courses Following Curative-Intent Resection for Intrahepatic Cholangiocarcinoma. Ann Surg Oncol. 2019; 26:2549-57.

28. Zhang XF, Beal EW, Bagante $F$, et al. Early versus late recurrence of intrahepatic cholangiocarcinoma after resection with curative intent. Br J Surg. 2018; 105: 848-56.

29. Wang $\mathrm{L}$, Ke $\mathrm{O}$, Lin $\mathrm{N}$, et al. Does postoperative adjuvant transarterial chemoembolization benefit for all patients with hepatocellular carcinoma combined with microvascular invasion: a meta-analysis. Scand J Gastroenterol. 2019; 54: 528-37.

30. Zhang XP, Liu YC, Chen $\mathrm{ZH}$, et al. Postoperative Adjuvant Transarterial Chemoembolization Improves Outcomes of Hepatocellular Carcinoma Associated with Hepatic Vein Invasion: A Propensity Score Matching Analysis. Ann Surg Oncol. 2019; 26: 1465-73.

31. Wang Z, Ren Z, Chen Y, et al. Adjuvant Transarterial Chemoembolization for HBV-Related Hepatocellular Carcinoma After Resection: A Randomized Controlled Study. Clin Cancer Res. 2018; 24: 2074-81.

32. Hatano E, Uemoto $S$, Yamaue $H$, et al. Significance of hepatic resection and adjuvant hepatic arterial infusion chemotherapy for hepatocellular carcinoma with portal vein tumor thrombus in the first branch of portal vein and the main portal trunk: a project study for hepatic surgery of the Japanese Society of Hepato-Biliary-Pancreatic Surgery. J Hepatobiliary Pancreat Sci. 2018; 25: $395-402$ 